\title{
Rotational stability of plasma blobs
}

\author{
D. A. D'Ippolito, J. R. Myra, and D. A. Russell \\ Lodestar Research Corporation, 2400 Central Avenue, Boulder, Colorado 80301 \\ G. Q. Yu \\ University of California, San Diego, California
}

March, 2004

(submitted to Physics of Plasmas) 


\title{
Rotational stability of plasma blobs*
}

\author{
D. A. D’Ippolito, ${ }^{\dagger}$ J. R. Myra, and D. A. Russell \\ Lodestar Research Corporation, 2400 Central Avenue, Boulder, Colorado 80301 \\ G. Q. Yu \\ University of California, San Diego, California
}

\begin{abstract}
The theory of plasma blobs is extended to treat the stability of "non-thermalized" blobs, which have both density and temperature higher than the surrounding plasma and can transport heat as well as particles. It is shown that the internal blob temperature profile $\mathrm{T}_{\mathrm{e}}(\mathrm{r})$ can drive azimuthal rotation $\mathrm{v}_{\theta}(\mathrm{r})$ about the blob axis, which produces a robust $\mathrm{m}=2$ rotational instability in the interchange limit $\left(\mathrm{k}_{\|}=0\right)$, similar to those considered earlier for rotating theta pinch and mirror plasmas. The instability includes the effects of the centrifugal and Coriolis forces, the sheared velocity $v_{\theta}(r)$, and the axial sheath boundary condition. In some parameter regimes, the growth rate can be large $\left(\gamma \tau_{\mathrm{c}}\right.$ $>>1$, where $\tau_{\mathrm{c}}$ is a typical blob radial convection time), and the rotational instability can play a role in determining the blob size distribution and radial transport. Rotation is expected to play a role in the evolution of blobs created by Edge Localized Modes (ELMs). Numerical calculations show that finite-Larmor-radius stabilization is ineffective for comparable ion and electron temperatures, but the sheath conductivity can be strongly stabilizing for reasonable parameters. A separate branch of temperaturegradient-driven sheath instabilities, predicted in the eikonal limit, is not observed for low mode numbers.
\end{abstract}

PACS numbers: 52.25.Fi, 52.35.Ra, 52.55.Dy, 52.55.Fa

†email: dasd@lodestar.com 


\section{Introduction}

There is growing experimental evidence ${ }^{1-13}$ from both linear and toroidal plasma devices that high-density plasma filaments carry a significant flux of particles across the scrape-off-layer (SOL) to the wall. The filaments are spatially extended along the magnetic field, but look like "blobs" in the plane perpendicular to B. A theoretical model ${ }^{14-17}$ treating the blobs as coherent, propagating objects shows that they become charge-polarized and acquire an induced electric field $\mathrm{E}_{\mathrm{y}}$ in the presence of a radial force $\mathrm{F}_{\mathrm{X}}$. The blobs convect via the $\mathbf{E} \times \mathbf{B}$ drift in the direction of the force, e.g. down the magnetic field gradient in a toroidal device such as a tokamak. (For a toroidal device, our local coordinates $\mathrm{x}$ and $\mathrm{y}$ refer to the $\mathrm{R}$ and $\mathrm{Z}$ directions; for a rotating linear machine, $\mathrm{x}$ and $y$ refer to $r$ and $\theta$. Also, $\mathrm{z}$ denotes the coordinate along the magnetic field.) This mechanism is analogous to the Rosenbluth-Longmire picture of interchange instability ${ }^{18}$ and is similar to the mechanism ${ }^{19}$ that propels high-field-side pellets into the core plasma. The convective transport of particles and energy by blobs is a good candidate mechanism to explain the intermittency in space and time observed in SOL turbulence measurements, as discussed in earlier papers. ${ }^{14-17}$ In a tokamak fusion reactor, blob transport could reduce the divertor efficiency and increase the wall interaction, producing a higher level of impurities. There is an active experimental and theoretical research effort underway to understand the properties of blobs and to quantify the associated transport.

In the simplest version of the blob model (appropriate for the far SOL), the electron temperature is assumed to be constant in space, and the model evolves only the density $n$ and potential $\varphi$. Thus, the blob is assumed to be in thermal equilibrium with the background plasma (no internal temperature gradients in $\mathrm{x}, \mathrm{y}$ ) and to be fully connected to the sheaths at the end of the field line (no $\mathrm{z}$ dependence). If the vorticity and background density are assumed to be small, a blob density of the form $n(x, y)=n(x) \exp \left(-y^{2} / 2 y_{b}{ }^{2}\right)$ yields a solution of the equations with a constant radial velocity, 14,15

$$
\mathrm{u}_{\mathrm{x}}=\frac{\Lambda}{\mathrm{y}_{\mathrm{b}}^{2}}
$$


where $y_{b}$ is the poloidal scale length of the blob (i.e. the distance over which charge polarization occurs), $\Lambda$ is a parameter that measures the strength of the outwards force relative to the sheath conductivity, and the dimensionless variables of Ref. 15 are used (velocity, length, and time are normalized to the sound speed $c_{s}$, gyroradius $\rho_{\mathrm{s}}=\mathrm{c}_{\mathrm{s}} / \Omega_{\mathrm{i}}$, and $\Omega_{\mathrm{i}}^{-1}$, respectively). For example, when the outwards force is provided by the curvature (and radial gradient) of the toroidal magnetic field in a tokamak, one finds that $\Lambda \equiv \mathrm{L}_{\|} / \mathrm{R}=\mathrm{q}$, where $\mathrm{L}_{\|}$is the parallel connection length and $\mathrm{R}$ is the major radius of the torus. In general, a force producing an outwards acceleration $\mathrm{a}_{\mathrm{x}}$ has $\Lambda=\mathrm{L}_{\|} \mathrm{a}_{\mathrm{x}}$. In what follows, we restrict the discussion to cylindrical blobs of radius $a=\mathrm{x}_{\mathrm{b}}=\mathrm{y}_{\mathrm{b}}$.

An important prediction of this simple blob model is that the outwards convective velocity $u_{\mathrm{x}}$ of the blob depends (through the charge polarization mechanism) on the blob size $a$, so that smaller blobs move faster than larger ones. Taking an ensemble average over blob sizes, one can show that the profiles of density $\mathrm{n}(\mathrm{x})$ and particle flux $\Gamma(\mathrm{x})=\mathrm{nu}_{\mathrm{x}}$ depend on the blob size distribution and contain a strong convective component in the far SOL when the smaller blobs are dominant. 15

The force responsible for the blob motion also drives internal or "secondary" instabilities 20,21 that fragment the blobs and thereby affect the ensemble-averaged blob transport (also see the review paper in Ref. 17). For large blobs, the curvature-driven sheath-interchange mode is the dominant instability; it causes blobs to bifurcate as they move across the SOL. ${ }^{20,21}$ For small blobs, the predominant instability is the KelvinHelmholtz mode due to the sheared velocity $\mathrm{v}_{\mathrm{x}}(\mathrm{y}) ;^{21}$ this mode causes the outer parts of the blobs to peel off and eventually destroys the coherent structure. The blobs which contribute to particle transport are those which are "effectively stable" on the time to transport across the SOL, ${ }^{20}$ i.e. blobs with $\gamma<1 / \tau_{\mathrm{w}}$, where $\gamma$ is the growth rate of the most unstable secondary mode and $\tau_{\mathrm{w}}=\Delta \mathrm{x}_{\mathrm{SOL}} / \mathrm{u}_{\mathrm{x}}$ is the blob convection time across the SOL. If we estimate the most stable blob radius $a_{*}$ (in the thermalized blob limit) by balancing the inertial, sheath and curvature terms in the vorticity equation, ${ }^{21}$ we obtain

$$
a_{*}=\left(\frac{\Lambda}{\alpha}\right)^{1 / 5}
$$


where $\Lambda=\mathrm{q}$ for the curvature force and $\alpha=2 \rho_{\mathrm{S}} / \mathrm{R}$ is the sheath conductivity parameter. For DIII-D parameters, this estimate yields $a \approx 15$ gyroradii corresponding to a blob size of about $1 \mathrm{~cm}$.

The present paper extends previous studies of blob stability to include "nonthermalized" blobs 22 which are hotter than the surrounding plasma and therefore have an internal electron temperature profile $\mathrm{T}_{\mathrm{e}}(\mathrm{r})$. This model is important for understanding blob dynamics at the edge-SOL interface. A blob is "born" near the separatrix as a result of the nonlinear development of small-scale turbulence ${ }^{5-13}$ or meso-scale instabilities such as Edge Localized Modes (ELMs). ${ }^{23-25}$ The blob carries with it the density and temperature of its place of origin, so that the core of the blob is both denser and hotter than the surrounding background plasma. The loss of heat along the field lines is much more rapid than the particle loss, so the blob quickly cools by parallel losses and comes into collisional thermal equilibrium with its surroundings. However, there is a region in the SOL near the separatrix where the blob retains a centrally-peaked temperature profile $\mathrm{T}_{\mathrm{e}}(\mathrm{r})$. In this paper, we assume for simplicity that the temperature is constant along the field lines, so that the blob is fully-connected to the sheaths and has the maximum Bohm potential. (In the disconnected limit, the temperature drops near the end plates and the sheath potential is greatly reduced.) Here, the dimensionless Bohm sheath potential is given by $\Phi_{\mathrm{B}}(\mathrm{r})=\mathrm{C}_{\mathrm{B}} \mathrm{T}$ with $\mathrm{C}_{\mathrm{B}}=\ln \left[\left(\mathrm{m}_{\mathrm{i}} / 2 \pi \mathrm{m}_{\mathrm{e}}\right)^{1 / 2}\right] \approx 3$, where we let $\mathrm{e} \Phi_{\mathrm{B}} / \mathrm{T}_{\mathrm{es}} \rightarrow \Phi_{\mathrm{B}}$ and $\mathrm{T}_{\mathrm{e}} / \mathrm{T}_{\mathrm{es}} \rightarrow \mathrm{T}$, as discussed after Eq. (11). Since $\mathrm{dT}_{\mathrm{e}} / \mathrm{dr}<0$, the sheath potential produces an outwards-pointing (positive) radial electric field $\mathrm{E}_{\mathrm{r}}$ and the resulting $\mathbf{E} \times \mathbf{B}$ drift causes the blob to rotate about its axis with a sheared velocity $v_{\theta}(r)$.

The dynamics of hot blobs and their interaction with external velocity shear layers has been discussed in detail elsewhere. ${ }^{22}$ The internal spin or rotation of the blob around its axis modifies both its radial velocity (by hindering the charge polarization ${ }^{22}$ ) and its stability characteristics. In the limit of rapid spin, the curvature-driven modes are suppressed $^{22}$ and but we show here that new rotational modes arise which can go unstable through a combination of centrifugal force, Coriolis, Kelvin-Helmholtz and 
rotational shear effects. When the sheath conductivity is included in the analysis, the stability equation also includes the Berk-Ryutov-Tsidulko (BRT) $\nabla \mathrm{T}_{\mathrm{e}}$-driven instabilities $^{26}$ in the eikonal limit. In the low mode number limit of interest here, the sheath term turns out to have a net stabilizing effect. These effects, driven by the blob's internal temperature gradient, are the subject of the present paper.

We are interested here in secondary instabilities of the blob itself, driven by the blob's internal profiles, not global rotational instabilities of the main plasma column. The latter instabilities are also interesting as a possible blob generation mechanism for rapidly rotating linear machines, but are not the subject of the present paper. The same formalism used for global instabilities in rotating theta pinches and mirror machines 27,28 can be applied in both problems.

We will show that blobs with azimuthal mode number $\mathrm{m}=2$ and $\mathrm{k}_{\|}=0$ are unstable to rotational modes when (i) $\mathrm{T}_{\mathrm{i}} / \mathrm{T}_{\mathrm{e}}$ is less than an FLR stabilization threshold of about 1020 and (ii) the sheath parameter $\mathrm{S}_{\alpha} \equiv \alpha a^{2} / \Omega_{\mathrm{p}}<1$, where $\Omega_{\mathrm{p}} \sim \mathrm{C}_{\mathrm{B}} / a^{2}$ is the rotation frequency at the radial location where the eigenfunction peaks. Here $S_{\alpha}$ denotes the ratio of the sheath conductivity term to the vorticity convection term driven by the rotation. Physically, the condition $\mathrm{S}_{\alpha}<1$ implies that the blob spin time around its axis $\left(\tau_{\mathrm{s}} \sim \Omega^{-1}\right)$ is shorter than the time for its vorticity to dissipate by parallel loss $\left(\tau_{\phi} \sim 1 / \alpha a^{2}\right) .15$ The condition $\mathrm{S}_{\alpha}<1$ is typically fulfilled for the intermediate-size blobs $\left(a=a_{*}>>1\right)$ which are the least unstable to curvature-driven and velocity-shear modes. Using Eq. (2) with $\Lambda=\mathrm{q}$, the condition $a=a_{*}$ implies that $\mathrm{S}_{\alpha} \sim \alpha a_{*}{ }^{4} / \mathrm{C}_{\mathrm{B}} \sim \mathrm{q} /\left(a_{*} \mathrm{C}_{\mathrm{B}}\right)<<1$. Thus, the sheath conductivity is not strong enough to stabilize the rotational instability for typical SOL parameters and blob sizes. In Sec.II, we will also show that the condition for the rotational growth rate to be larger than other secondary instability growth rates for a blob of radius $a$ and azimuthal mode number $\mathrm{m}$ [see Eq. (7)] is $\mathrm{m}<\left(\mathrm{C}_{\mathrm{B}} a / \mathrm{q}\right)^{1 / 2}$. This relation shows that the rotational mode dominates for low mode numbers and large blobs.

The plan of this paper is the following. In Sec. II we give a more detailed comparison of the scaling of the blob rotational growth rate with the growth rates of the instabilities that are dominant in the absence of blob spin. In Sec. III it is shown that the 
well-known rotational stability equation can be derived from the blob model equations. In Sec. IV, the results of a 1D linear stability analysis and 2D nonlinear simulation code are presented for the case $T_{i}=0$. The influence of finite-Larmor-radius (FLR) effects for $T_{i} \neq$ 0 and sheath effects are also discussed. Finally, a brief summary and discussion is given in Sec. V.

\section{Scaling of instability growth rates}

To put the present work in context, we begin by comparing the scaling of the growth rate $\gamma_{R}$ of the rotational instability with those of secondary instabilities that control the evolution of blobs in the thermalized (non-rotating) limit: the sheathinterchange mode 20,21 with growth rate $\gamma_{\mathrm{SI}}$ and the Kelvin-Helmholtz $(\mathrm{KH})$ mode 21 driven by the linear blob motion $v_{\mathrm{X}}(\mathrm{y})$ with growth rate $\gamma_{\mathrm{KH}}$. The relative scaling of the growth rates determines the parameter regime in which the rotational mode dominates.

Here, we take into account only the internal temperature profile $\mathrm{T}_{\mathrm{e}}(\mathrm{r})$ of the blobs and neglect the temperature variation $\mathrm{T}_{\mathrm{e}}(\mathrm{x})$ of the external background plasma, assuming for simplicity that the latter varies on a scale length much longer than the blob radius $a$. For a hot blob, the radially decaying sheath potential implies a radial electric field with $\mathrm{E}_{\mathrm{r}}$ $>0$, which causes the blob to rotate in the azimuthal or $\theta$ direction with an angular frequency $\Omega(\mathrm{r})=\mathrm{v}_{\theta}(\mathrm{r}) / \mathrm{r}$. The resulting centrifugal, Coriolis, and velocity-shear effects can drive low azimuthal-mode-number instabilities with eigenfunctions varying like $\psi(r) e^{\operatorname{im} \theta}$. Here, $(r, \theta, z)$ are used to represent the cylindrical blob coordinates and no variation in the axial coordinate $\mathrm{z}$ (parallel to $\mathbf{B}$ ) is assumed in either the equilibrium or stability analysis.

The blob stability can be described by the $\mathrm{B}_{\theta}=0, \mathrm{k}_{\|}=0, \beta_{\mathrm{t}}=8 \pi \mathrm{p} / \mathrm{B}^{2}=0$ limits of the Freidberg-Pearlstein theory 27 developed for theta-pinches. In Ref. 27, a radial differential equation (discussed in the next section) and an associated variational principle are given which include rotational, finite-Larmor radius (FLR), and line bending $\left(\mathrm{k}_{\|} \neq 0\right)$ effects. For present purposes, we examine the scaling of the driving term 
in the variational principle. Balancing this term against the inertial term gives the following (dimensional) scaling of the growth rate:

$$
\gamma^{2} \approx\left(\frac{\mathrm{k}_{\theta}}{\mathrm{k}_{\perp}}\right)^{2}\left(\frac{\mathrm{a}}{\mathrm{L}_{\mathrm{n}}}\right) \Omega \Omega_{\mathrm{E}} .
$$

Here $\mathrm{k}_{\theta}=\mathrm{m} / \mathrm{r}, \Omega=\mathrm{v}_{\theta} / \mathrm{r}$ is the angular rotation frequency, $\Omega_{\mathrm{E}}=-\mathrm{cE}_{\mathrm{r}} / \mathrm{rB} \approx(\mathrm{c} / \mathrm{rB})\left(\mathrm{d} \Phi_{\mathrm{B}} / \mathrm{dr}\right)$ is the $\mathbf{E} \times \mathbf{B}$ rotation frequency, and $\Phi_{\mathrm{B}} \approx 3 \mathrm{~T}_{\mathrm{e}}$. In the presence of FLR effects $\left[\Omega_{*} \equiv\right.$ $\left.-\mathrm{c} /(\mathrm{ernB})\left(\mathrm{dp}_{\mathrm{i}} / \mathrm{dr}\right) \neq 0\right]$, the angular frequencies are related by the equilibrium radial force balance condition:

$$
\Omega=\Omega_{\mathrm{E}}-\Omega_{*},
$$

where $\Phi=\Omega_{*} / \Omega_{\mathrm{E}} \sim \mathrm{T}_{\mathrm{i}} / \mathrm{T}_{\mathrm{e}}$ is the FLR parameter. Since $\mathrm{E}_{\mathrm{r}}>0$ and $\mathrm{dp}_{\mathrm{i}} / \mathrm{dr}<0$ for the blob, both terms in Eq. (4) have the same sign and the diamagnetic drift increases the instability drive.

In addition to the diamagnetic drift contribution to equilibrium force balance contained in Eq. (4), there is also an explicit FLR stabilization term $\left(\propto \Omega_{*}{ }^{2}\right)$ in the variational principle, which is neglected in Eq. (3). The effect of FLR on stability will be included in the solution of the radial stability equation in Sec. IV. For now, we anticipate that result and point out that FLR stabilization requires that the $\varpi^{2}$ term be larger than the (destabilizing) $\Phi$ term, which requires $\Phi>>1$. Thus, FLR stabilization of blobs is more difficult than in the well-known theta pinch problem ${ }^{27}$ for which $\Phi \sim 1$ is sufficient to obtain stability.

Neglecting $\Omega_{*}$ and assuming that the sheath potential decays over a blob radius $a$, we can estimate the magnitude of the rotation and the growth rate. Setting $\mathrm{L}_{\mathrm{n}} \sim \mathrm{L}_{\mathrm{T}} \sim a$ and $\mathrm{k}_{\perp} \sim \mathrm{k}_{\theta}$, the rotational growth rate $\gamma_{\mathrm{R}}$ has the following dimensional scaling:

$$
\gamma_{\mathrm{R}} \sim \Omega \sim\left(\frac{\rho_{\mathrm{s}}}{\mathrm{a}}\right)^{2} \Omega_{\mathrm{i}} .
$$

In the dimensionless notation of Ref. 15, Eq. (5) becomes $\gamma_{\mathrm{R}} \sim 1 / a^{2}$. 
Using results from previous papers, 20,21 the comparison with the sheathinterchange growth rate $\gamma_{\mathrm{SI}}$ and the Kelvin-Helmholtz $(\mathrm{KH})$ growth rate $\gamma_{\mathrm{KH}}$ is

$$
\gamma_{\mathrm{R}} \sim \frac{\mathrm{C}_{\mathrm{B}}}{\mathrm{a}^{2}}, \gamma_{\mathrm{SI}} \sim \frac{\mathrm{m}^{2} \mathrm{q}}{\mathrm{a}^{3}}, \gamma_{\mathrm{KH}} \sim \frac{\mathrm{q}}{\mathrm{a}^{3}}
$$

where $\mathrm{C}_{\mathrm{B}}$ is the Bohm sheath coefficient defined earlier. The $\mathrm{KH}$ mode referenced in Eq. (6) is a global (low-m) mode driven by the curvature-driven sheared flow, $\mathrm{v}_{\mathrm{x}}(\mathrm{y})$, for a blob propagating radially across a background plasma, ${ }^{21}$ and the growth rate is estimated as $\gamma \sim \mathrm{v}_{\mathrm{x}} / \mathrm{L}_{\mathrm{y}} \sim \Lambda / a^{3}$ using Eq. (1). There is also a KH instability associated with the rotation, which has a growth rate $\gamma \sim \mathrm{v}_{\theta} / \mathrm{r} \sim \Omega \sim \mathrm{C}_{\mathrm{B}} / a^{2}$, comparable to the mode driven by the centrifugal force. The numerical solutions of Sec. IV include the effects of the azimuthal rotational shear $v_{\theta}(r)$. For the remainder of this paper, we will not distinguish between the centrifugal and velocity-shear drives associated with rotation and will simply refer to both of these as rotational instabilities.

The main conclusion to draw from Eq. (6) is that the rotational growth rate is dominant for larger blobs and lower mode numbers $\mathrm{m}$. Comparing the rotational and sheath-interchange growth rates for a fixed blob size, we find that $\gamma_{R}>\gamma_{S I}$ for

$$
\mathrm{m}<\mathrm{m}_{\text {crit }} \sim\left(\mathrm{C}_{\mathrm{B}} \mathrm{a} / \mathrm{q}\right)^{1 / 2}
$$

which is also the condition to neglect the curvature term in the vorticity equation. Finally, we point out that the convection rate ${ }^{20}$ of blobs across the SOL scales like $\gamma_{\mathrm{c}} \sim 1 / a^{3}$ using Eq. (1), so that convection is also slow compared to rotational instability growth for typical blobs which are much larger than the gyroradius $(a>>1)$.

\section{Derivation of rotational stability equation}

In this section we show that the well-known rotational stability equation without FLR stabilization can be recovered from the basic equations of blob theory. The generalization to obtain the FLR terms is obtained heuristically. 


\section{A. Basic Equations}

The theory of propagating density blobs and their stability is based on a simple set of dimensionless equations that expresses the conservation of charges and particles. ${ }^{14,15}$ Here, we include the spatial variation of the electron temperature, so that the starting point of our analysis is based on the vorticity, continuity, and temperature equations: ${ }^{15}$

$$
\begin{aligned}
& \nabla \cdot\left(\mathrm{n} \frac{\mathrm{d}}{\mathrm{dt}} \nabla_{\perp} \Phi\right)=\alpha \mathrm{nT} \mathrm{T}^{-1 / 2}\left(\Phi-\Phi_{\mathrm{B}}\right)-\beta \nabla_{\mathrm{y}}(\mathrm{nT}), \\
& \frac{\mathrm{dn}}{\mathrm{dt}}+\alpha \mathrm{T}^{1 / 2} \mathrm{n}=0 \\
& \frac{\mathrm{dT}}{\mathrm{dt}}+\alpha_{\mathrm{T}} \mathrm{T}^{3 / 2}=0 \\
& \frac{\mathrm{d}}{\mathrm{dt}}=\frac{\partial}{\partial \mathrm{t}}+\mathbf{v} \cdot \nabla
\end{aligned}
$$

where $\Phi$ is the total electrostatic potential, $\Phi_{\mathrm{B}}$ is the Bohm sheath potential, and the first term on the right-hand-side (rhs) of the vorticity equation was expanded in the limit $\varphi=$ $\Phi-\Phi_{B} \ll 1$. Also, $n$ is the particle density $\left(n=n_{e}=n_{i}\right), T=T_{e}$ is the electron temperature, $\alpha=\left(2 \rho_{\mathrm{s}} / \mathrm{L}_{\|}\right)$measures the net parallel current and particle loss into the sheaths, $\alpha_{\mathrm{T}}$ gives the energy loss into the sheaths, and $\beta=\left(2 \rho_{\mathrm{S}} / \mathrm{R}\right)$ is the curvature parameter. Consistent with the blob model, we assume that the velocity is given by the $\mathbf{E} \times \mathbf{B}$ drift, $\mathbf{v}=\mathbf{b} \times \nabla \Phi=\mathbf{e}_{\mathrm{z}} \times \nabla \Phi$. To make the equations dimensionless, time has been normalized to $\Omega_{\mathrm{i}}^{-1}$, length scales to $\rho_{\mathrm{s}}$, and other quantities to reference values (e.g. separatrix values $\mathrm{n}_{\mathrm{s}}$ and $\mathrm{T}_{\mathrm{es}}$ ) so that $\Omega_{\mathrm{i}} \mathrm{dt} \rightarrow \mathrm{dt}, \rho_{\mathrm{s}} \nabla \rightarrow \nabla$, e $\varphi / \mathrm{T}_{\mathrm{es}} \rightarrow \varphi, \mathrm{v} / \mathrm{c}_{\mathrm{s}} \rightarrow \mathrm{v}, \mathrm{n} / \mathrm{n}_{\mathrm{s}}$ $\rightarrow \mathrm{n}$, and $\mathrm{T}_{\mathrm{e}} / \mathrm{T}_{\mathrm{es}} \rightarrow \mathrm{T}$, where $\Omega_{\mathrm{i}}=\mathrm{eB} / \mathrm{m}_{\mathrm{i}} \mathrm{c}$ is the ion cyclotron frequency and $\rho_{\mathrm{s}}=\mathrm{c}_{\mathrm{s}} / \Omega_{\mathrm{i}}$ is the (constant) gyroradius based on $\mathrm{c}_{\mathrm{s}}=\left(\mathrm{T}_{\mathrm{es}} / \mathrm{m}_{\mathrm{i}}\right)^{1 / 2}$.

We make the standard assumptions that the plasma filaments are localized perpendicular to $\mathbf{B}$ and employ a slab model with orthogonal coordinates $(\mathrm{x}, \mathrm{y}, \mathrm{z})$ to represent the tokamak geometry. The $\mathrm{x}$ coordinate is taken in the direction of the major radius $R$, and the dimensionless curvature is written as $\kappa=-\left(\rho_{s} / R\right) \hat{\mathbf{e}}_{\mathrm{x}}$. The $\mathrm{z}$ coordinate is 
taken along the direction of $\mathbf{B}$, so that $2 \mathbf{b} \times \kappa \cdot \nabla=-\beta \nabla_{\mathrm{y}}$ and $\mathrm{y}$ is approximately in the poloidal direction at the outer midplane. Here, $y=0$ denotes the location of the outer midplane and $\mathrm{x}>0$ corresponds to the SOL, so that motion in the positive $\mathrm{x}$ direction is outwards towards the wall. For the stability analysis in Sec. III B, we also introduce the corresponding cylindrical blob coordinate system $(\mathrm{r}, \theta, \mathrm{z})$.

For the rotational stability analysis, it is useful to make a number of simplifying assumptions. We restrict the discussion to time scales smaller than the parallel loss times, $\mathrm{t} \ll<\alpha_{\mathrm{T}}^{-1}<<\alpha^{-1}$, so that we can neglect the rhs of Eqs. (9) and (10). This restricts the validity of the stability analysis to growth rates satisfying $\gamma \sim \Omega>>\alpha_{\mathrm{T}}$. With this approximation, we observe that the temperature equation (10) can be solved by the ansatz:

$$
\mathrm{T}=\mathrm{n}^{\mathrm{v}}
$$

We also neglect the curvature term $(\propto \beta)$ in Eq. (8), as detailed studies of curvaturedriven blob instabilities have already been published 20,21 and we would like to study the properties of instabilities driven only by rotational physics. The neglect of curvature is justified for large blobs satisfying Eq. (7).

Using these approximations and combining Eqs. (8) and (9), we obtain the following set of reduced equations as a starting point for the rotational stability analysis:

$$
\begin{gathered}
\nabla \cdot\left(\mathrm{n} \frac{\mathrm{d}}{\mathrm{dt}} \nabla_{\perp} \Phi\right)=\alpha \mathrm{nT}^{-1 / 2}\left(\Phi-\Phi_{\mathrm{B}}\right), \\
\frac{\mathrm{dn}}{\mathrm{dt}}=0
\end{gathered}
$$

When Eq. (13) is linearized, the inertial term on the left-hand-side (lhs) contains the drive terms for the centrifugal, Coriolis, and Kelvin-Helmholtz effects. The sheath term on the rhs yields the drive term for the BRT electron-temperature-gradient instability in the eikonal limit. ${ }^{26}$ However, we will show that the sheath term turns out to have a net stabilizing effect in the low mode number limit of interest here. 


\section{B. Perturbation Analysis}

We assume a 1D blob equilibrium (varying only in $r$ ) and linearize the equations (13) and (14) using the following ansatz:

$$
\begin{aligned}
& n=\bar{n}(r)+\tilde{n}(r) e^{i m \theta} e^{-i \omega t}, \\
& \varphi=\bar{\varphi}(r)+\tilde{\varphi}(r) e^{i m \theta} e^{-i \omega t},
\end{aligned}
$$

where $\bar{\varphi}=\Phi_{B}(r)$. Linearizing Eq. (14) leads to the following solution for $\tilde{n}$

$$
\tilde{\mathrm{n}}=-\frac{\mathrm{m}}{\mathrm{r}} \frac{\overline{\mathrm{n}}^{\prime}(\mathrm{r})}{\omega-\mathrm{m} \Omega} \tilde{\varphi}
$$

For the moment, we neglect the sheath term $(\alpha \rightarrow 0)$ on the rhs of Eq. (13) in order to derive the usual magnetohydrodynamic (MHD) result; we will add the linearized sheath term later to obtain the final stability equation. The linearized form of the lhs of Eq. (13) can be written as

$$
\nabla \cdot \tilde{\mathbf{X}}=0, \quad \tilde{\mathbf{X}}=\overline{\mathrm{D}}_{\mathrm{t}}\left(\overline{\mathrm{n}} \nabla_{\perp} \tilde{\varphi}\right)+\overline{\mathrm{D}}_{\mathrm{t}}\left(\tilde{\mathrm{n}} \nabla_{\perp} \bar{\varphi}\right)+\tilde{\mathrm{D}}_{\mathrm{t}}\left(\overline{\mathrm{n}} \nabla_{\perp} \bar{\varphi}\right),
$$

where $D_{t}=d / d t$, as defined in Eq. (11) with $\mathbf{v}=\mathbf{b} \times \nabla \varphi$. After some algebra, it can be shown that

$$
\begin{gathered}
\tilde{\mathrm{X}}_{\mathrm{r}}=-\mathrm{i}(\omega-\mathrm{m} \Omega) \overline{\mathrm{n}} \widetilde{\varphi}^{\prime}-\mathrm{im}(\overline{\mathrm{n}} \Omega)^{\prime} \widetilde{\varphi}-\frac{2 \mathrm{im}}{\mathrm{r}} \overline{\mathrm{n}} \Omega \widetilde{\varphi}-\mathrm{i} \Omega(\omega-\mathrm{m} \Omega) \mathrm{r} \tilde{\mathrm{n}}, \\
\tilde{\mathrm{X}}_{\theta}=\frac{\mathrm{m}}{\mathrm{r}}(\omega-\mathrm{m} \Omega) \overline{\mathrm{n}} \tilde{\varphi}+2 \Omega \overline{\mathrm{n}} \widetilde{\varphi}^{\prime}+\Omega^{2} \mathrm{r} \tilde{\mathrm{n}} .
\end{gathered}
$$

Next we substitute Eq. (16) for $\tilde{\mathrm{n}}$ into the expressions for the components of $\tilde{\mathbf{X}}$ and make the transformation $\tilde{\varphi} \rightarrow \psi$, defined by

$$
\widetilde{\varphi}=(\omega-\mathrm{m} \Omega) \psi
$$


The quantity $\psi$ is related to the radial component of the plasma displacement $\xi$, and this transformation is necessary to put the eigenmode equation into the same form as in Ref. 27. Combining Eqs. (16) and (20) gives

$$
\tilde{\mathrm{n}}=-\frac{\mathrm{m}}{\mathrm{r}} \bar{n}^{\prime} \psi
$$

Carrying out the transformation of Eqs. (18) and (19) using Eqs. (20) and (21), we obtain

$$
\begin{gathered}
i \tilde{X}_{r}=(\omega-m \Omega)^{2} \bar{n} \psi^{\prime}+2 m \Omega(\omega-m \Omega) \bar{n} \frac{\psi}{r}, \\
\tilde{X}_{\theta}=\frac{m}{r}(\omega-m \Omega)^{2} \bar{n} \psi-m\left(\bar{n} \Omega^{2}\right)^{\prime} \psi+2 \Omega(\omega-m \Omega) \bar{n} \psi^{\prime},
\end{gathered}
$$

which are substituted into the equation

$$
\mathrm{i} \nabla \cdot \tilde{\mathrm{X}}=\frac{1}{\mathrm{r}} \frac{\partial}{\partial \mathrm{r}} \mathrm{r}\left(\mathrm{i} \tilde{\mathrm{X}}_{\mathrm{r}}\right)-\frac{\mathrm{m}}{\mathrm{r}} \tilde{\mathrm{X}}_{\theta}=0
$$

The result can be put into the following form

$$
\frac{\mathrm{d}}{\mathrm{dr}} \mathrm{r} \overline{\mathrm{n}} \mathrm{F} \frac{\mathrm{d} \psi}{\mathrm{dr}}-\mathrm{m}^{2} \overline{\mathrm{n}} \mathrm{F} \frac{\psi}{\mathrm{r}}-\frac{\mathrm{d}}{\mathrm{dr}}\left[\overline{\mathrm{n}}\left(\mathrm{F}-\omega^{2}\right)\right] \psi=0
$$

where $F \equiv(\omega-m \Omega)^{2}$ and $F-\omega^{2}=m \Omega(m \Omega-2 \omega)$.

In order to compare Eq. (25) with the result of Ref. 27, a relation is needed between $\psi$ and $\xi$. This follows from the definition of the perturbed velocity

$$
\widetilde{\mathbf{v}} \equiv \frac{\mathrm{d} \xi}{\mathrm{dt}}=-\mathrm{i}\left(\omega-\mathrm{k}_{\theta} \mathrm{v}_{\theta}\right) \xi=-\mathrm{i}(\omega-\mathrm{m} \Omega) \xi
$$

and its identification with the $\mathbf{E} \times \mathbf{B}$ drift, $\widetilde{\mathbf{v}}=\hat{\mathbf{e}}_{\mathrm{z}} \times \nabla \widetilde{\varphi}$. Taking the radial component of Eq. (26), we obtain

$$
\widetilde{\mathrm{v}}_{\mathrm{r}} \equiv \frac{\mathrm{d} \xi}{\mathrm{dt}}=-\mathrm{i}(\omega-\mathrm{m} \Omega) \xi
$$

whereas the explicit calculation from the $\mathbf{E} \times \mathbf{B}$ drift using the transformation (20) gives 


$$
\widetilde{\mathrm{v}}_{\mathrm{r}}=-\mathrm{ik}_{\theta} \tilde{\varphi}=-\mathrm{i} \frac{\mathrm{m}}{\mathrm{r}}(\omega-\mathrm{m} \Omega) \psi
$$

where $\xi=\xi_{\mathrm{r}}$ and $\mathrm{k}_{\theta}=\mathrm{m} / \mathrm{r}$. Equating the two expressions for $\tilde{\mathrm{v}}_{\mathrm{r}}$ gives the desired relation between $\psi$ and $\xi$ :

$$
\psi=\frac{\mathrm{r} \xi}{\mathrm{m}}
$$

Note that combining Eqs. (21) and (29) recovers the usual MHD result that $\tilde{\mathrm{n}}=-\xi \cdot \nabla \overline{\mathrm{n}}$. Inserting Eq. (29) into Eq. (25), we obtain

$$
\frac{d}{d r} r \bar{n} F \frac{d(r \xi)}{d r}-m^{2} \bar{n} F \xi-\frac{d}{d r}\left[\bar{n}\left(F-\omega^{2}\right)\right] r \xi=0
$$

After some straightforward algebra, it can be shown that Eq. (30) is equivalent to the form given in the original paper 27

$$
\frac{d}{d r} r^{3} n F \frac{d \xi}{d r}-\left(m^{2}-1\right) n F r \xi+\frac{d\left(n \omega^{2}\right)}{d r} r^{2} \xi=0
$$

To summarize the results of this section, we have provided a new derivation of a well-known result, obtaining the rotational stability equation of magnetohydrodynamics (MHD) theory in the limits $\mathrm{B}_{\theta}=0, \mathrm{k}_{\|}=0$, and $\beta_{\mathrm{t}}=0$ from a particular limit of the blob model equations. This derivation gives us confidence that we can apply the usual rotational instability analysis 27 to the blob equilibrium solution. Finite gyroradius effects can be included heuristically by using the form of the function F obtained previously 27 in the limit $\Omega_{*} \neq 0$, viz.

$$
\mathrm{F}(\mathrm{r}, \omega)=(\omega-\mathrm{m} \Omega)\left(\omega-\mathrm{m} \Omega_{\mathrm{E}}\right)
$$

It is also straightforward to add the sheath conductivity term [ $\alpha$ term in Eq. (8)] to the derivation. If one linearizes the sheath term retaining temperature perturbations, $\tilde{\mathrm{T}}=-\xi \cdot \nabla \overline{\mathrm{T}}$, there is a cancellation of the rotational drift terms and the stability equation becomes 


$$
r \frac{d}{d r} r \bar{n} F \frac{d \psi}{d r}-m^{2} \bar{n} F \psi-r \frac{d}{d r}\left[\bar{n}\left(F-\omega^{2}\right)\right] \psi-\frac{i \alpha \bar{n} \omega r^{2}}{\bar{T}^{1 / 2}} \psi=0
$$

In the rest of the paper, we drop the overbars on all equilibrium quantities.

Finally, we examine the dimensionless parameters for the stability problem. The blob radius $a$ can be scaled out of the system of equations (32) and (33) with the rotation frequency represented by $\Omega_{\mathrm{p}} \sim \mathrm{C}_{\mathrm{B}} / a^{2}$, the rotation frequency at the radial location where the eigenfunction peaks. The system is then characterized by the three dimensionless parameters $\mathrm{m}, \bar{\sigma}=\Omega_{*} / \Omega_{\mathrm{E}} \sim \mathrm{T}_{\mathrm{i}} / \mathrm{T}_{\mathrm{e}}$, and $\mathrm{S}_{\alpha}=\left(\alpha a^{2}\right) / \Omega_{\mathrm{p}}$ characterizing the mode width, the FLR physics and the sheath conductivity, respectively.

\section{Blob Stability Results}

\section{A. Blob Equilibrium}

We now apply the formalism of Sec. III to an equilibrium solution for rotating blobs. The normalized blob density profile $n(r)$ is specified as

$$
n(r)=(1-\varepsilon) e^{-r^{2} /\left(2 a^{2}\right)}+\varepsilon
$$

where the total density (blob + background) at $r=0$ is normalized to unity. The background is assumed to consist of a constant "floor" density $n_{f}$ and $\varepsilon=n_{f} /\left(n_{b}+n_{f}\right)$, where $n_{b}$ is the peak blob density. We set $\varepsilon=0.01$ to ensure a localized solution for the blob potential;20 the effects of varying the ratio $\varepsilon$ on the blob velocity and stability were explored in Ref. 20 and will not be repeated here. The function T(r) given in Eq. (12), T $=[n(r)]^{\nu}$, determines both the internal electron and ion temperature profiles for the blob, $\mathrm{T}_{\mathrm{e}}=\mathrm{T}_{\mathrm{e} 0} \mathrm{~T}(\mathrm{r})$ and $\mathrm{T}_{\mathrm{i}}=\mathrm{T}_{\mathrm{i} 0} \mathrm{~T}(\mathrm{r})$. As discussed in Sec. I, we assume $\mathrm{T}=$ const. along the field lines so that the blobs are fully connected to the sheaths and the Bohm sheath potential is given by $\Phi_{B}(r)=C_{B} T(r)$, where $C_{B}=\ln \left[\left(m_{i} / 2 \pi m_{e}\right)^{1 / 2}\right] \approx 3$. The blob polarization potential, $\varphi_{\text {pol }}(\mathrm{y})=\Lambda \nabla_{\mathrm{y}} \ln \mathrm{n}(\mathrm{y}), 14,15$ which drives the radial motion in Eq. (1), is smaller than the Bohm potential $\left(\varphi_{\text {pol }} / \Phi_{\mathrm{B}} \sim \mathrm{r}_{\mathrm{S}} / a<<1\right)$ and can be neglected in the stability analysis and in computing the rotation. The rotation frequency $\Omega(\mathrm{r})$ is determined by radial force balance including both the $\mathbf{E} \times \mathbf{B}$ and diamagnetic drifts [see 
Eq. (4)]. For the choice of profiles in Eqs. (12) and (34), the rotational frequencies $\Omega_{\mathrm{E}}$, $\Omega_{*}$, and $\Omega$ are proportional to $\mathrm{T}(\mathrm{r})$ in the limit $\varepsilon<<1$.

In Fig. 1, the profiles $\mathrm{n}(\mathrm{r} / a)$ and $\Omega(\mathrm{r} / a)$ are shown for the base case $(\mathrm{v}=2)$. Note that the rotation profile is far from the familiar rigid-rotor limit $(\Omega=$ const) which is often invoked in studying rotational stability. Thus, in addition to purely centrifugal and Coriolis effects, angular-velocity-shear will also play a role in determining the blob stability. This effect can be stabilizing or destabilizing (Kelvin-Helmholtz instability) depending on the parameters. We will return to this point later.

\section{B. Linear Stability Analysis}

The eigenfunction $\psi(\mathrm{r})$ is obtained by solving Eq. (33) with $\mathrm{F}(\mathrm{r}, \omega)$ defined by Eq. (32), subject to the boundary conditions that $\psi^{\prime} / \psi=\mathrm{m} / \mathrm{r}$ as $\mathrm{r} \rightarrow 0$ and $\psi^{\prime} / \psi=-\mathrm{m} / \mathrm{r}$ as $\mathrm{r}$ $\rightarrow \infty$, where $\psi^{\prime}=\mathrm{d} \psi / \mathrm{dr}$. The results presented here were obtained using a shooting method to determine the eigenvalue $\omega$ by requiring that the logarithmic derivative of $\psi$ be continuous at an interior matching point. The shooting code has been checked using another code employing a matrix method and by comparison with an analytic solution for a sharp-boundary density, rigid-rotor profile.

First, we consider blob stability in the absence of FLR effects $\left(\mathrm{T}_{\mathrm{i} 0}=0\right)$ and sheath conductivity $(\alpha=0)$. In Fig. 2 we show a plot of the density profile $n / n_{0}$, the rotational mode instability drive $\left[\propto\left(\mathrm{r}^{2} / \mathrm{n}\right) \mathrm{d}(\mathrm{n} \Omega) / \mathrm{dr}\right]$, and the amplitude of the $\mathrm{m}=2$ eigenfunction $|\psi| \mathrm{vs} r / a$ for the case $a=10$ (in units of $\rho_{\mathrm{s}}$ ), $\varepsilon=0.01$, and $v=2$. Each profile is normalized to have a maximum value of approximately unity to illustrate the relative shapes. Note that the $m=2$ rotational mode eigenfunction $|\psi|(r)$ peaks off axis in the lowdensity outer region of the blob $(1<\mathrm{r} / \mathrm{a}<2)$ where the drive term maximizes.

Consistent with the $\mathrm{k}_{\|}=0$ limit of earlier work, we find that the $\mathrm{m}=1$ rotational mode is marginally stable. ${ }^{27}$ For the present parameters, the rotational modes with $m \geq 3$ are stable. Although most $\mathrm{m}$ modes are stable, we will show that the $\mathrm{m}=2$ mode is robustly unstable, at least in the absence of sheath effects.

In Fig. 3, we show the effect of the FLR terms. We compare the stability of the $\mathrm{m}$ $=2$ mode for temperature profiles of different widths $(v=0.5,1.0$, and 2.0) and include 
the effect of the FLR terms $\left(\mathrm{T}_{\mathrm{i} 0} / \mathrm{T}_{\mathrm{e} 0} \neq 0\right)$. For each value of $v$, we plot the normalized growth rate $\gamma a^{2}$ vs $\mathrm{T}_{\mathrm{i} 0} / \mathrm{T}_{\mathrm{e} 0}$ for a case with small background density $(\varepsilon=0.01)$ and without the sheath conductivity term $(\alpha=0)$. As discussed in Sec. II, the rotational growth rate has the scaling that $\gamma a^{2}$ is independent of $a$; thus, the growth rate curves in Fig. 3 are valid for all values of the blob radius. The figure shows that the instability growth rate increases with the temperature gradient (larger $v$ ), and the FLR stabilization is weak for all the temperature profiles considered with $\mathrm{T}_{\mathrm{i} 0} / \mathrm{T}_{\mathrm{e} 0}<10$. The lack of stabilization is explained by the discussion after Eq. (4), where it was pointed out that the $\Omega_{\mathrm{E}}$ and $\Omega_{*}$ terms add to give a large destabilizing rotation. Hence, the rotational mode growth rate $\gamma$ increases with $\varpi=\Omega_{*} / \Omega_{\mathrm{E}} \sim \mathrm{T}_{\mathrm{i}} / \mathrm{T}_{\mathrm{e}}$ and has a maximum for $\varpi$ of order unity. FLR stabilization requires $\Phi>>1$ in order that the destabilizing $\Phi$ term be smaller than the stabilizing $\varpi^{2}$ term. This property of the rotating blob differs from that of theta pinches for which the $\Omega_{\mathrm{E}}$ and $\Omega_{*}$ terms tended to cancel, making them easier to stabilize by FLR effects.

Next, we consider the effect of the sheath boundary condition on the stability of low-m rotational modes. In Fig. 4 , we show the $m=2$ normalized growth rate $\gamma a^{2}$ vs the sheath conductivity parameter $S_{\alpha}=\alpha a^{2} / \Omega_{p}$ for the base case temperature profile $(v=$ 2.0) with $\varepsilon=0.01$ and $\mathrm{T}_{\mathrm{i} 0} / \mathrm{T}_{\mathrm{e} 0}=0$. Here, $\Omega_{\mathrm{p}} \sim \mathrm{C}_{\mathrm{B}} / a^{2}$ is the value of the rotation frequency at the radius $r$ where the eigenfunction peaks, and the parameter $S_{\alpha}$ specifies the ratio of the sheath conductivity to the vorticity associated with the rotation. The decrease in growth rate with $S_{\alpha}$ illustrates the stabilizing effect of the sheath conductivity. It was difficult to find numerical solutions for $S_{\alpha} \geq 1$ using the shooting algorithm because the eigenfunctions become very localized and the mode eventually stabilizes. Physically, the condition $S_{\alpha}<<1$ implies that the blob spin time around its axis $\left(\tau_{\mathrm{s}} \sim \Omega^{-1}\right)$ is much shorter than the time for its vorticity to dissipate by parallel loss $\left(\tau_{\phi} \sim 1 / \alpha a^{2}\right)$ [see Ref. 15, Eq. (32)]. Since the rotational instability is driven by the lhs of the vorticity equation, the parallel loss of vorticity can stabilize the mode when $S_{\alpha}=$ $\alpha a^{2} / \Omega_{\mathrm{p}}>1$. 
In the eikonal or high-m limit $\left(\mathrm{k}_{\mathrm{r}} \sim \mathrm{k}_{\theta}=\mathrm{m} / \mathrm{r}>>1 / a\right)$, one can show that Eq. (33) yields the following well-known local dispersion relation 26,29

$$
\left(\omega-\omega_{E}\right)^{2}+i \omega_{s} \omega=0
$$

where $\omega_{\mathrm{E}} \equiv \mathrm{m} \Omega_{\mathrm{E}} \rightarrow \mathrm{m} \Omega$ in the limit $\mathrm{T}_{\mathrm{i}} \rightarrow 0$ and $\omega_{\mathrm{s}}=\alpha / \mathrm{k}_{\perp}^{2} \rightarrow \alpha \mathrm{r}^{2} / \mathrm{m}^{2}$ for $\mathrm{k}_{\mathrm{r}}<<\mathrm{k}_{\theta}$. This dispersion relation predicts unstable BRT modes driven by the internal temperaturegradient in the limit of high sheath conductivity $\left(\mathrm{S}_{\alpha}\right)$. However, more detailed analytic and numerical investigations show that the blob' s cylindrical geometry, together with monotonically decreasing T(r) profiles, typically prevents the existence of a "radial well" necessary for localized absolutely-unstable modes in the eikonal limit. Physically, this may be related to the fact that the sheath drive $\omega_{\mathrm{s}}$ vanishes at $\mathrm{r}=0$ where the rotation $\Omega$ (proportional to the temperature-gradient) maximizes.

For $\mathrm{S}_{\alpha}<<1$ and $\mathrm{T}_{\mathrm{i} 0} / \mathrm{T}_{\mathrm{e} 0}<10$, the growth rate of the $\mathrm{m}=2$ rotational instability is large enough that it can have important implications for blob transport. Table I shows the normalized complex eigenfrequency $\omega a^{2}$ for three temperature profiles using the parameters $\varepsilon=0.01, \mathrm{~T}_{\mathrm{i} 0} / \mathrm{T}_{\mathrm{e} 0}=0$ and $\alpha=0$. Both the real and imaginary parts of the eigenvalue increase with the temperature gradient parameter $v$. For the three profiles considered, we find the universal result that $\gamma a^{2}$ is order unity (independent of the blob radius $a$ ). Since $a$ is measured here in units of the gyroradius, $a \gg 1$ for the typical blobs observed in experiments, and $\gamma a^{2} \sim 1$ implies that $\gamma a^{3}>>1$. This means that the instability growth time $\tau=\gamma^{-1}$ is short compared to the time for the blob to convect one blob radius, $\tau_{\mathrm{c}}=a / \mathrm{u}_{\mathrm{x}} \sim a^{3}$. Thus, the rotational modes are expected to impact the blob transport. The extent of their effect depends on the details of the eigenfunction; Fig. 2 shows that the eigenfunction affects mainly the outer portion of the blob, so we expect that the main effect of the rotational instability will be to cause the blob to shed some of its density in the outer mantel. This is illustrated by the $2 \mathrm{D}$ simulation discussed in the next section. 


\section{Table I}

$\mathrm{m}=2$ eigenfrequency for parameters $a=10, \varepsilon=0.01, \mathrm{~T}_{\mathrm{i} 0} / \mathrm{T}_{\mathrm{e} 0}=0$ and $\alpha=0$

\begin{tabular}{|c|c|c|c|}
\hline & $v=0.5$ & $v=1.0$ & $v=2.0$ \\
\hline$\omega a^{2}$ & $-0.96+0.57 \mathrm{i}$ & $-1.6+0.76 \mathrm{i}$ & $-2.6+0.94 \mathrm{i}$ \\
\hline
\end{tabular}

\section{2D Simulation}

In this section, we complement the linear 1D theory presented in Sec. IV B by presenting results of a 2D nonlinear simulation of blob transport that solves the following equations

$$
\begin{gathered}
\nabla \cdot\left(\mathrm{n} \frac{\mathrm{d}}{\mathrm{dt}} \nabla_{\perp} \Phi\right)=\alpha \mathrm{n}\left(\Phi-\Phi_{\mathrm{B}}\right)-\beta \nabla_{\mathrm{y}} \mathrm{n}, \\
\frac{\mathrm{dn}}{\mathrm{dt}}+\alpha \mathrm{n}=0,
\end{gathered}
$$

with $\Phi_{\mathrm{B}}=\Phi_{\mathrm{B} 0} \mathrm{n}$. All quantities are assumed to be constant along the field lines. The code used was a slight generalization of the one employed in a previous study of blob instabilities, ${ }^{21}$ in that the Bohm sheath term $\left(\alpha n \Phi_{B}\right)$ was added on the rhs of the vorticity equation to drive the rotation. Note that the $2 \mathrm{D}$ code includes the rotational, curvature and sheath conductivity terms but neglects FLR effects.

The 2D simulation including the Bohm sheath effects shows good agreement with the 1D stability results described in the previous section. This is illustrated by a snapshot shown in Fig. 5 of a case with parameters $a=10, \alpha=3 \times 10^{-5}, \beta=6.9 \times 10^{-4}$ and $\Phi_{\mathrm{B} 0}$ $=10$, so that $\mathrm{S}_{\alpha} \approx \alpha a^{4} / \Phi_{\mathrm{B} 0}=0.03$. The blob develops an $\mathrm{m}=2, \mathrm{k}_{\|}=0$ instability on a timescale short compared to the theoretical convection time $\tau_{\mathrm{c}}=a / \mathrm{u}_{\mathrm{x}}$, i.e. $\gamma a^{3}>>1$, in agreement with the linear stability analysis. The frame in Fig. 5 occurs after about one $\tau_{\mathrm{c}}$ and shows that the instability has undergone several e-foldings to produce an observable distortion of the blob. The instability peaks near the outside of the blob, as predicted by the linear stability analysis, and throws off an outer shell of material that wraps around to 
form a "halo" or "tail". The tail is left behind by the blob' s rotation and propagation to the right. The instability shown here is clearly rotational in origin and differs qualitatively from the curvature-driven blob instabilities ${ }^{20,21}$ studied earlier. The dominance of the rotational instability in this run is consistent with the conditions $S_{\alpha}<1$ and $m<\left(C_{B}\right.$ $a / q)^{1 / 2}$ discussed in previous sections. A similar run with $a=30$, implying $S_{\alpha}=2.4$, was also unstable, but the instability had a different character (not shown here). This is in agreement with the present theory, where $S_{\alpha} \geq 1$ implies strong stabilization of the rotational branch.

We remark in passing that the blob dynamics (e.g. temporal variation of $\mathrm{v}_{\mathrm{x}}$ and $\mathrm{v}_{\mathrm{y}}$ ) at later times is very interesting in the simulation corresponding to Fig. 5, as other rotational effects 22 come into play. For example, the radial velocity of the blob is observed to increase after throwing off its outer mantel and it develops a poloidal velocity. A complete description of the simulation results will be presented elsewhere. ${ }^{30}$

\section{Summary and Discussion}

In this paper, we have studied the rotational stability of non-thermalized blobs, for which the interior is hotter than the surrounding plasma. The study of temperature profile effects in blobs is motivated by recent observations that ELMs produce blob-like transport.9,23-25 Large ELMs are typically associated with high-confinement H-modes, which have hot pedestals and SOL plasmas which are attached to the divertor plates. Under these conditions, the blobs produced by the ELM crashes are expected to have substantial size and to transport energy as well as particles.

In the "fully-connected" limit discussed here, the blob profiles are assumed to be constant along the field line, extending to the sheaths at the divertor plates (or other boundary surface). In this limit, the internal blob electron temperature profile $\mathrm{T}_{\mathrm{e}}(\mathrm{r})$ produces a Bohm sheath potential $\Phi_{\mathrm{B}}(\mathrm{r})=\mathrm{C}_{\mathrm{B}} \mathrm{T}_{\mathrm{e}}(\mathrm{r}) \approx 3 \mathrm{~T}_{\mathrm{e}}(\mathrm{r})$ and radial electric field $\mathrm{E}_{\mathrm{r}}=$ $-\nabla_{\mathrm{r}} \Phi_{\mathrm{B}}$ so that the blob spins about its axis. This rotation influences the blob transport in several ways. In a separate paper, 22 it is shown that the rotation can inhibit the charge polarization mechanism, thereby reducing the blob radial velocity and suppressing blob 
instabilities 20 driven by curvature. In the present paper, we have shown that new instabilities arise in spinning blobs, driven by the centrifugal and Coriolis forces and by angular-velocity-shear. These instabilities cause the blob to shed density from its outer mantel and to deform in shape. The residual density thrown off from the spinning blob is left behind and does not contribute to transport. A quantitative assessment of the consequence of the rotational instability on the blob transport is beyond the scope of this paper. However, the present work, together with Ref. 22, show the importance of internal temperature gradient effects on the blob dynamics.

In the interchange $\left(\mathrm{k}_{\|}=0\right)$ limit studied in this paper, the only unstable azimuthal mode has $\mathrm{m}=2$. The $\mathrm{m}=1$ mode is marginally stable for typical profiles, and modes with $m \geq 3$ are stable, even neglecting FLR effects and sheath conductivity. Based on previous work, ${ }^{27}$ we expect that blobs with structure along the field line $\left(\mathrm{k}_{\|} \neq 0\right)$ might also be unstable to $m=1$ modes. We have shown that FLR stabilization is weak for $T_{i} / T_{e}$ $\sim 1$ because $\mathrm{E}_{\mathrm{r}}<0$ in the hot blobs, so that the $\mathbf{E} \times \mathbf{B}$ drift and diamagnetic drift reinforce one another rather than cancel. However, sheath conductivity stabilizes the rotational modes when $\mathrm{S}_{\alpha}=\alpha a^{2} / \Omega_{\mathrm{p}}>1$ where $\Omega_{\mathrm{p}}$ is the rotational frequency $\Omega \sim \mathrm{C}_{\mathrm{B}} / a^{2}$ evaluated at the location where the eigenfunction peaks. The condition for rotational instability to dominate the curvature and sheath effects can be expressed as $a \sim a_{*}<<a_{\mathrm{sh}}$, where $a_{\mathrm{sh}}=$ $\left(\mathrm{C}_{\mathrm{B}} / \alpha\right)^{1 / 4}$.

Nonlinear 2D simulations show qualitative agreement with the linear stability analysis. Further analysis of the simulations is needed to establish the effect of the rotational instabilities on the overall blob transport.

\section{Acknowledgements}

This work was supported by the U.S. Department of Energy (DOE) under DOE Grants No. DE-FG03-97ER54392 and DE-FG03-00ER54568; however, this support does not constitute an endorsement by the DOE of the views expressed herein. 


\section{References}

${ }^{1}$ S. J. Zweben, Phys. Fluids 28, 974 (1985).

${ }^{2}$ M. Endler, H. Niedermeyer, L. Giannone, E. Holzhauer, A. Rudyj, G. Theimer, N. Tsois and ASDEX team, Nucl. Fusion 35, 1307 (1995).

${ }^{3}$ R. A. Moyer, R. D. Lehmer, T. E. Evans, R. W. Conn, and L. Schmitz, Plasma Phys. Contr. Fusion 38, 1273 (1996).

${ }^{4}$ M. Umansky, S. I. Krasheninnikov, B. LaBombard and J. L. Terry, Phys. Plasmas 5, 3373 (1998); B. LaBombard, M. V. Umansky, R. L. Boivin, J. A. Goetz, J. Hughes, B. Lipschultz, D. Mossessian, C. S. Pitcher, J. L. Terry, Alcator Group, Nucl. Fusion 40, 2041 (2000).

${ }^{5}$ G. Y. Antar, P. Devynck, X. Garbet, and S. C. Luckhardt, Phys. Plasmas 8, 1612 (2001).

${ }^{6}$ G. Y. Antar, S. I. Krasheninnikov, P. Devynck, R. P. Doerner, E. M. Hollmann, J. A. Boedo, S. C. Luckhardt and R. W. Conn, Phys. Rev. Letters 87, 065001 (2001).

${ }^{7}$ J. Boedo, D. Rudakov, R. Moyer, et al., Phys. Plasmas 8, 4826 (2001).

${ }^{8}$ S. Zweben, D. P. Stotler, J. L. Terry, et al., Phys. Plasmas 9, 1981 (2002).

${ }^{9}$ D. L. Rudakov, J. A. Boedo, R. A. Moyer, et al., Plasma Phys. Contr. Fusion 44, 717 (2002).

${ }^{10}$ J. Neuhauser, D. Coster, H. U. Fahrbach, et al., Plasma Phys. Contr. Fusion 44, 855 (2002).

${ }^{11}$ J. L. Terry, S. J. Zweben, K. Hallatschek, et al., Phys. Plasmas 10, 1739 (2003).

12 J. A. Boedo, D. L. Rudakov, R. A. Moyer, et al., Phys. Plasmas 10, 1670 (2003).

13 "High speed imaging of edge turbulence in NSTX," S. Zweben, R. Maqueda, D.P. Stotler, et al., to be published in Nucl. Fusion (2003).

${ }^{14}$ S. I. Krasheninnikov, Phys. Lett. A 283, 368 (2001).

${ }^{15}$ D. A. D'Ippolito, J. R. Myra, and S. I. Krasheninnikov, Phys. Plasmas 9, 222 (2002).

${ }^{16}$ S. I. Krasheninnikov and A. I. Smolyakov, Phys. Plasmas 10, 3020 (2003). 
${ }^{17}$ D. A. D'Ippolito, J. R. Myra, S. I. Krasheninnikov, G. Q. Yu, and A. Yu. Pigarov, Invited Paper, 2003 PET meeting, San Diego, CA, Sept. 3-5, 2003; to be published in Contrib. Plasma Phys 44 (2004).

${ }^{18}$ M. N. Rosenbluth and C. L. Longmire, Ann. Phys. (N.Y.) 1, 120 (1957);

${ }^{19}$ P. Parks, W. D. Sessions, and L. R. Baylor, Phys. Plasmas 7, 1968 (2000).

${ }^{20}$ D. A. D'Ippolito and J. R. Myra, Phys. Plasmas 10, 4029 (2003).

${ }^{21}$ G. Q. Yu and S. I. Krasheninnikov, Phys. Plasmas 10, 4413 (2003).

22 J. R. Myra, D. A. D'Ippolito, S. I. Krasheninnikov, and G. Q. Yu, Lodestar Research Corporation report \#LRC-04-98 (2004), to be submitted for publication.

23 M. Endler, S. Davies, I. Garcia-Cortes et al., in Proceedings of the 29th EPS Conference on Plasma Phys. and Control. Fusion, Montreux, Switzerland, 2002, edited by R.Behn and C. Varandas (European Physical Society, Paris, 2002), Europhysics Conference Abstracts Vol. 26B, paper O-3.24.

${ }^{24}$ B. Gonçalves, C. Hidalgo, M. A. Pedrosa et al., Plasma Phys. Control. Fusion 45, 1627 (2003).

${ }^{25}$ J. A. Boedo, D. L. Rudakov, R. A. Moyer et al., submitted to Phys. Plasmas (2003).

${ }^{26}$ H. L. Berk, D. D. Ryutov, and Yu. A. Tsidulko, Phys. Fluids B 3, 1346 (1991).

27 J. P. Freidberg and L. D. Pearlstein, Phys. Fluids 21, 1207 (1978).

28 J. P. Freidberg and D. A. D' Ippolito, Phys. Fluids26, 2657 (1983), and references therein.

${ }^{29}$ J. R. Myra, D. A. D'Ippolito, and J. P. Goedbloed, Phys. Plasmas 4, 1330 (1997).

${ }^{30}$ G. Q. Yu and S. I. Krasheninnikov, private communication (2004). 


\section{Figure Captions}

Fig. 1 Plots of the blob density profile n(r) (solid line) and the rotation frequency profile $|\Omega(\mathrm{r})|$ in arbitrary units (short dashed line), with all profiles normalized to have a maximum value of unity. The parameters are $a=10, \varepsilon=0.01, \mathrm{v}=2$, and $\mathrm{T}_{\mathrm{i} 0} / \mathrm{T}_{\mathrm{e} 0}$ $=0$, and $\alpha=0$.

Fig. 2 Plots of the blob density profile $n(r)$ (solid line), rotational drive $\left(r^{2} / n\right) d(n \Omega) / d r$ (long dashed line), and eigenfunction $|\psi(\mathrm{r})|$ (short dashed line), with all profiles normalized to have a maximum value of order unity. The parameters are $\mathrm{m}=2, a$ $=10, \varepsilon=0.01, v=2$, and $\mathrm{T}_{\mathrm{i} 0} / \mathrm{T}_{\mathrm{e} 0}=0$, and $\alpha=0$.

Fig. 3 Plot of the $\mathrm{m}=2$ normalized growth rate $\gamma a^{2}$ vs $\mathrm{T}_{\mathrm{i} 0} / \mathrm{T}_{\mathrm{e} 0}$ for three temperature profiles $(\nu=0.5,1.0$, and 2.0$)$ with $\varepsilon=0.01$ and $\alpha=0$. The instability growth rate increases with the temperature gradient (larger $v$ ), and FLR stabilization is weak for all temperature profiles when $\mathrm{T}_{\mathrm{i} 0} / \mathrm{T}_{\mathrm{e} 0}$ is order unity. Note that these are universal growth rate curves with respect to blob radius ( $\gamma a^{2}$ is independent of $a$ ).

Fig. 4 Plot of the $m=2$ normalized growth rate $\gamma a^{2}$ vs the sheath parameter $\alpha a^{2} / \Omega_{p}$ for the base case temperature profile $(\mathrm{v}=2.0)$ with $\varepsilon=0.01$ and $\mathrm{T}_{\mathrm{i} 0} / \mathrm{T}_{\mathrm{e} 0}=0$. The decrease in growth rate with $\alpha a^{2} / \Omega_{\mathrm{p}}$ illustrates the stabilizing effect of the sheath conductivity $\alpha$. Blob rotational effects are important when $\alpha a^{2} / \Omega_{\mathrm{p}}<1$, where $\Omega_{\mathrm{p}}$ is the value of the rotation frequency at the radius where the eigenfunction peaks.

Fig. 5 Snapshot of a spinning blob taken from a 2D simulation solving Eqs. (36) and (37) for the parameters $a=10, \alpha=3 \times 10^{-5}, \beta=6.9 \times 10^{-4}$ and $\Phi_{\mathrm{B} 0}=10$. This frame shows the development of an $\mathrm{m}=2$ instability with $\gamma \sim \Omega$. The instability peaks near the edge of the blob and throws out a thin mantle of plasma. At later times in the simulation, this mantle is left behind as the blob spins and translates to the right. 


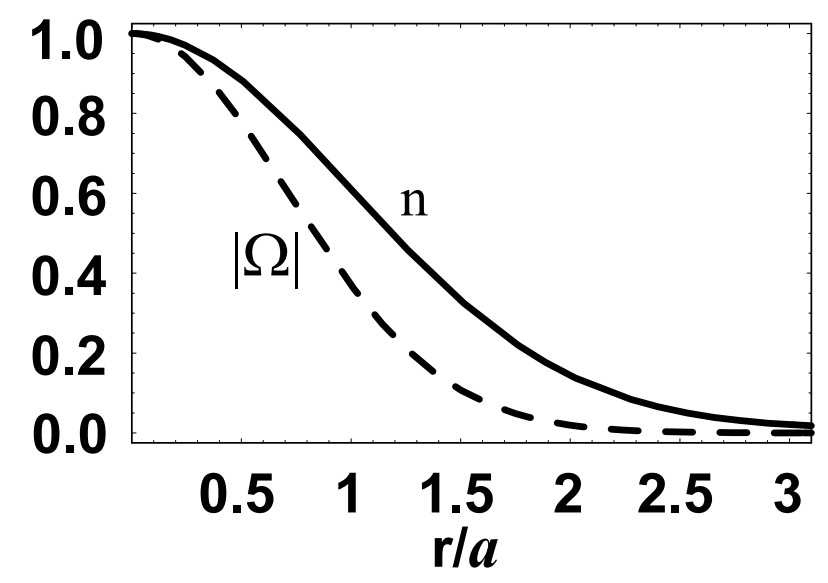

Fig. 1 Plots of the blob density profile $n(r)$ (solid line) and the rotation frequency profile $|\Omega(\mathrm{r})|$ in arbitrary units (short dashed line), with all profiles normalized to have a maximum value of unity. The parameters are $a=10, \varepsilon=0.01, \mathrm{v}=2$, and $\mathrm{T}_{\mathrm{i} 0} / \mathrm{T}_{\mathrm{e} 0}$ $=0$, and $\alpha=0$.

Fig 1 


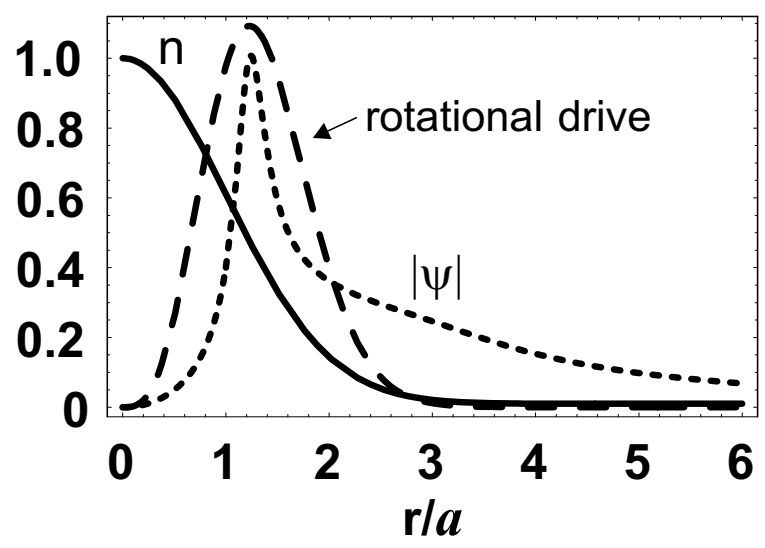

Fig. 2 Plots of the blob density profile $n(r)$ (solid line), rotational drive $\left(r^{2} / n\right) d(n \Omega) / d r$ (long dashed line), and eigenfunction $|\psi(\mathrm{r})|$ (short dashed line), with all profiles normalized to have a maximum value of order unity. The parameters are $\mathrm{m}=2, a$ $=10, \varepsilon=0.01, \nu=2$, and $\mathrm{T}_{\mathrm{i} 0} / \mathrm{T}_{\mathrm{e} 0}=0$, and $\alpha=0$.

Fig 2 


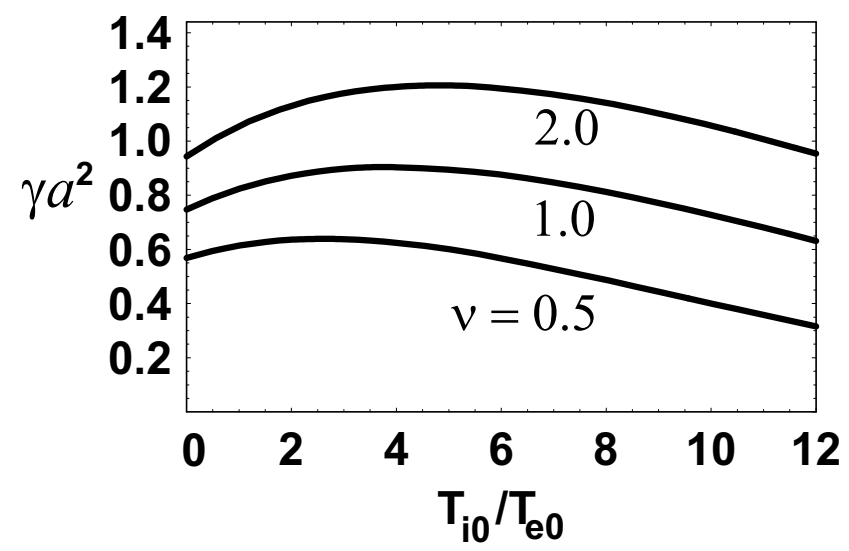

Fig. 3 Plot of the $\mathrm{m}=2$ normalized growth rate $\gamma a^{2} \mathrm{vs} \mathrm{T}_{\mathrm{i} 0} / \mathrm{T}_{\mathrm{e} 0}$ for three temperature profiles $(\nu=0.5,1.0$, and 2.0$)$ with $\varepsilon=0.01$ and $\alpha=0$. The instability growth rate increases with the temperature gradient (larger $v$ ), and FLR stabilization is weak for all temperature profiles when $\mathrm{T}_{\mathrm{i} 0} / \mathrm{T}_{\mathrm{e} 0}$ is order unity. Note that these are universal growth rate curves with respect to blob radius ( $\gamma a^{2}$ is independent of $a$ ).

Fig 3 


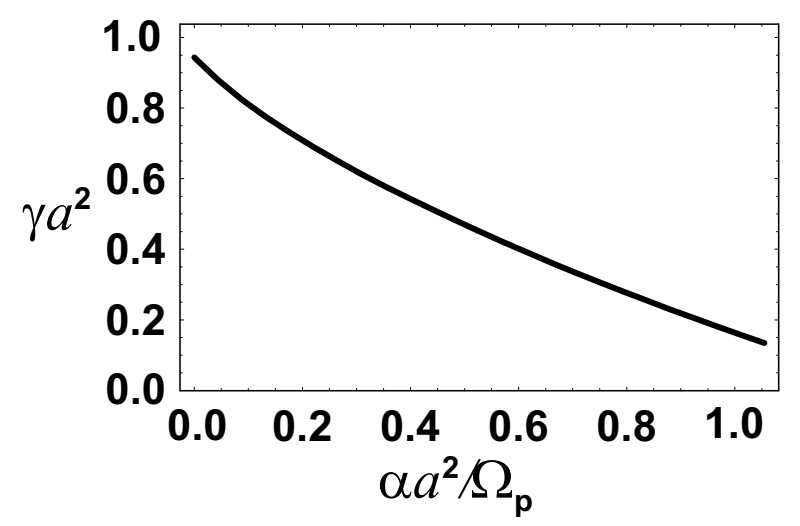

Fig. 4 Plot of the $\mathrm{m}=2$ normalized growth rate $\gamma a^{2}$ vs the sheath parameter $\alpha a^{2} / \Omega_{\mathrm{p}}$ for the base case temperature profile $(\mathrm{v}=2.0)$ with $\varepsilon=0.01$ and $\mathrm{T}_{\mathrm{i} 0} / \mathrm{T}_{\mathrm{e} 0}=0$. The decrease in growth rate with $\alpha a^{2} / \Omega_{\mathrm{p}}$ illustrates the stabilizing effect of the sheath conductivity $\alpha$. Blob rotational effects are important when $\alpha a^{2} / \Omega_{\mathrm{p}}<1$, where $\Omega_{\mathrm{p}}$ is the value of the rotation frequency where the eigenfunction peaks.

Fig 4 


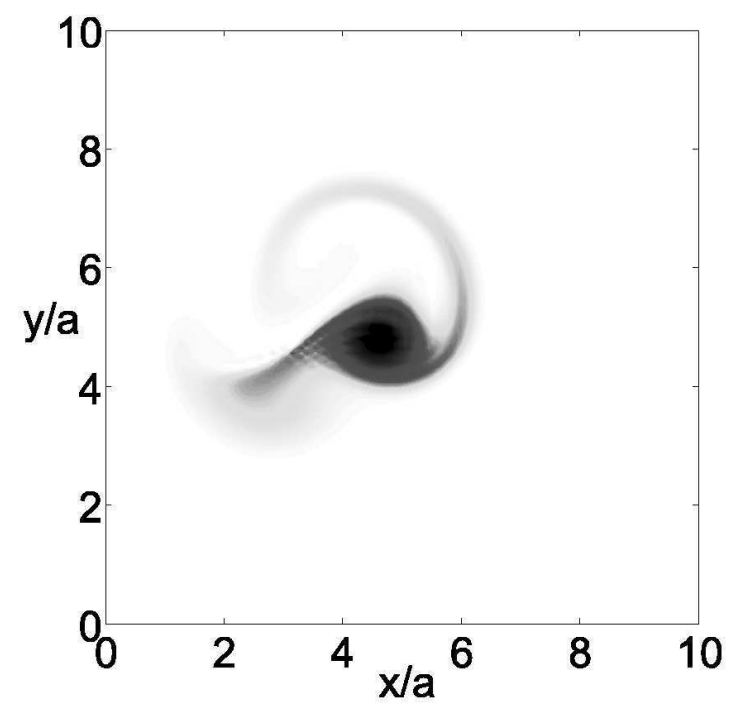

Fig. 5 Snapshot of a spinning blob taken from a 2D simulation solving Eqs. (36) and (37) for the parameters $a=10, \alpha=3 \times 10^{-5}, \beta=6.9 \times 10^{-4}$ and $\Phi_{\mathrm{B} 0}=10$. This frame shows the development of an $\mathrm{m}=2$ instability with $\gamma \sim \Omega$. The instability peaks near the edge of the blob and throws out a thin mantle of plasma. At later times in the simulation, this mantle is left behind as the blob spins and translates to the right.

Fig 5 